\title{
UTILIZAÇÃO DA TRANSFORMADA WAVELET COMPLEXA, AGRUPAMENTO E INTEGRAL FUZZY NO RECONHECIMENTO DE FACES HUMANAS
}

\author{
André Luiz Nogueira*, Junior Leal do Prado ${ }^{\dagger}$ \\ * Instituto Federal de Sergipe \\ Lagarto, Sergipe, Brasil \\ ${ }^{\dagger}$ Instituto Federal de Sergipe \\ Aracaju, Sergipe, Brasil
}

Emails: andreln27@yahoo.com, jrprado@gmail.com

\begin{abstract}
Resumo - Neste artigo, apresentamos um método para o reconhecimento de faces combinando a Transformada Wavelet Complexa, Análise de Componentes Principais (PCA), o Agrupamento Fuzzy e Integral Fuzzy. O método proposto é dividido basicamente em quatro etapas, em que a primeira utiliza a Transformada Wavelet Complexa para extração de características das faces. A segunda etapa consiste na aplicação da PCA às imagens obtidas anteriormente. Em seguida, é aplicado o Agrupamento Fuzzy que determinará os protótipos (centros) de cada classe e, por fim, na última etapa é utilizada a Integral Fuzzy, responsável pela fusão dos classificadores. Os resultados experimentais feitos utilizando-se imagens da base Yale e revelam que o algoritmo proposto apresentou um desempenho superior aos algoritmos que utilizam apenas uma das subimagens obtidas após a aplicação da Transformada Wavelet Complexa.
\end{abstract}

Keywords - agrupamento fuzzy, integral fuzzy, reconhecimento de padrões, transformada wavelet, PCA

\section{Introdução}

O reconhecimento de faces é uma subárea do reconhecimento de padrões, que vem recebendo uma atenção especial devido a suas aplicações comerciais, de segurança e militares.

Uma imagem de $q x p$ pixels pode ser representada por um vetor em um espaço de dimensão $q p$. Essa dimensão pode ser muito alta comprometendo a robustez e a velocidade do reconhecimento.

Um modo comum para resolver esse problema é utilizar técnicas para redução e seleção de características. Uma das mais populares é a Análise de Componentes Principais (Bruce A. Draper and Bevedge, 2003; Kyung-A Kim and Choi, 2004).

A PCA projeta os dados originais em um subespaço de dimensão reduzida, preservando a maior parte da informação original. Além da PCA, outra abordagem vem sendo empregada no reconhecimento de faces envolve a Transformada Wavelet (Goswami and Chan, 2011; Kyung-A Kim and Choi, 2004; Water, 1999). Nos trabalhos iniciais, a Transformada Wavelet era aplicada na compressão, redução de ruído e detecção de características de imagens. Recentemente, foi proposta a Transformada Wavelet Complexa de árvore dupla (TWC-AD) que supera a Transformada Wavelet (real), pois é aproximadamente invariante a deslocamentos e apresenta um desempenho superior na detecção de bordas de imagens (Liu and Dai, 2009; Ivan W. Selesnick and Kingsbury, 2005).

Atualmente, no reconhecimento de padrões, vem sendo utilizada a Análise de Agrupamentos Fuzzy (Bezdek, 1981; Bezdek and Dunn, 1975), cujo objetivo é classificar os dados de acordo com a similaridade entre eles e organizá-los em grupos.

Considerando as diferentes performances dos classificadores, é desejável que sua fusão produza classificadores com desempenhos melhores do que aqueles obtidos utilizando os classificadores individualmente. Baseado em Kwak and Pedrycz (2004), a Integral Fuzzy pode ser usada para fusão de classificadores.

O algoritmo proposto utiliza para extração de características das imagens a Transformada Wavelet Complexa e a PCA, já para a classificação as técnicas empregadas são Agrupamento Fuzzy e Integral Fuzzy.

Diante de uma revisão bibliográfica recentemente realizada, foi constatado que a fusão dessas abordagens supracitadas em um único algoritmo, visando ao reconhecimento de faces, ainda não foi muito explorada. Comprova-se esse fato devido a uma reduzida quantidade de publicações no meio científico que utilizam essas técnicas integradas. Nesse ínterim, o presente artigo vem contribuir com alguns passos, nesta direção, e evidenciar a superioridade do algoritmo proposto quando comparada aos classificadores individualmente.

\section{PCA}

A Análise de Componentes Principais (Bruce A. Draper and Bevedge, 2003; Kyung-A Kim and Choi, 2004; Martinez and Kak, 2001) é usada para determinar um subespaço, cujos vetores da base correspondem à direção de máxima variância no espaço original. Uma imagem de qxp pixels pode ser associada a um vetor de dimensão qp.

A PCA é capaz de transformar um espaço de dimensão $M$ em um espaço de dimensão $N$ $(M>>N)$, preservando a maior parte dos dados 
originais. O algoritmo PCA é descrito a seguir.

A base de vetores PCA é calculada a partir de um conjunto $I$, de imagens de treinamento. Primeiramente, as matrizes associadas às imagens são concatenadas formando um vetor e, feito isso, calcula-se a média desse conjunto. A seguir, subtrai-se a média de cada um dos vetores de $I$, obtendo $I_{1}, I_{2}, \ldots, I_{n}$.

Esses vetores são arranjados em uma matriz $\mathrm{X}$, um vetor por coluna.

$$
X=\left[I_{1}, I_{2}, \ldots, I_{n}\right] .
$$

onde, $M=X X^{T}$ é a matriz de covariância do conjunto de treinamento. Os componentes principais são calculados resolvendo a equação:

$$
R^{T}\left(X X^{T}\right) R=\Lambda \text {. }
$$

onde $\Lambda$, é a matriz diagonal de autovalores e $R$ é a matriz dos autovetores. O autovetor associado ao maior autovalor indica a característica de maior variância; o autovetor associado ao segundo maior autovalor é ortogonal ao primeiro e, assim sucessivamente. Os autovalores säo ordenados e apenas $N$ autovetores associados aos maiores autovalores são usados para definir o subespaço (PCA) que, consequentemente, terá dimensão $N$.

No algoritmo proposto, a PCA é executada nas subimagens obtidas após a aplicação da Transformada Wavelet Complexa.

\section{Transformada Wavelet Complexa}

A Transformada Wavelet Real (TWR) (KyungA Kim and Choi, 2004; P.C.Yuen and G.C.Feng, 1998) é uma poderosa ferramenta na resolução de problemas das ciências e engenharia. Entre eles podemos destacar: redução de ruído em sinais de áudio e imagens, compressão de sinais, detecção de objetos e reconhecimento de imagens. A TWR é sensível a oscilações e não é invariante a deslocamentos.

A Transformada Wavelet Complexa (TWC) (Liu and Dai, 2009; Ivan W. Selesnick and Kingsbury, 2005) surge como uma alternativa que supera a TWR, já que é menos sensível a oscilações e é aproximadamente invariante a deslocamentos.

Na figura 2, tem-se coeficientes obtidos após a aplicação da TWR na função apresentada na figura 1. Nota-se que no gráfico da figura 2, tem-se uma oscilação o que dificulta a localização de singularidades. Todavia, quando aplicamos a mesma função à TWC e geramos um gráfico com a magnitude dos coeficientes Wavelets, temos o gráfico da figura 3 , cujo ponto de singularidade é bem mais fácil de ser determinado, já que o gráfico não apresenta oscilações.

$\mathrm{Na}$ figura 4, tem-se, na primeira linha, dois gráficos da mesma função: um deslocado em relação ao outro. Os gráficos da segunda linha foram obtidos aplicando a TWR nas funções da primeira linha. Pode ser observado que esses gráficos são diferentes, ou seja, representações diferentes para a mesma função, o que não é desejável quando se está trabalhando com reconhecimento de padrões. Os gráficos na segunda linha da figura 5, foram obtidos aplicando a TWC. Nesse caso, observa-se que os gráficos são praticamente os mesmos, logo, um pequeno deslocamento não pertuba a representação do sinal após a aplicação da Transformada.

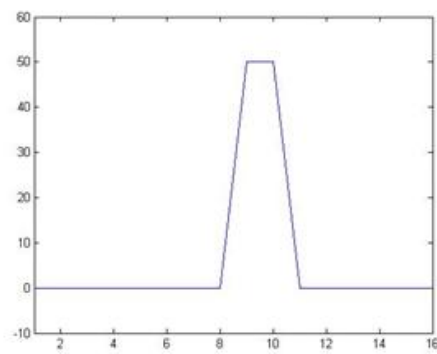

Fig. 1. Sinal Original

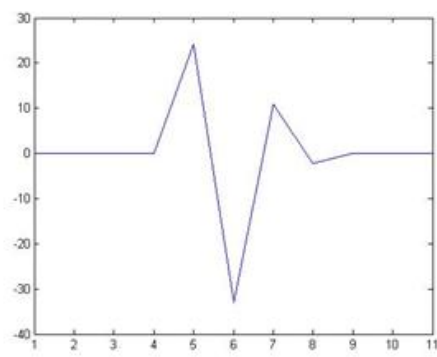

Fig. 2. Sinal obtido após aplicação da TWR

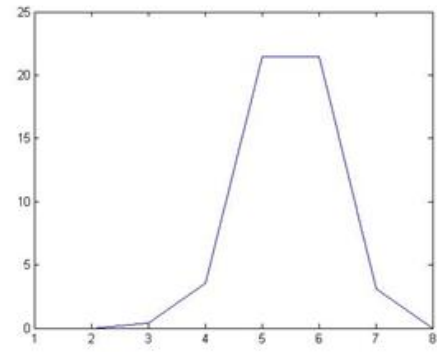

Fig. 3. Sinal obtido após aplicação da TWC
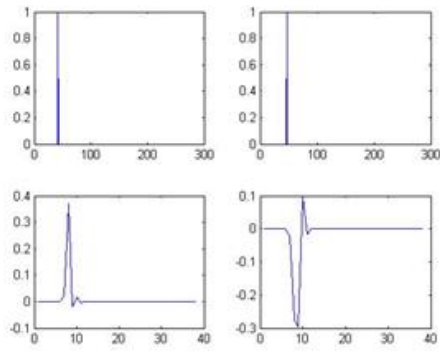

Fig. 4. Sinais Original (Originais), transladado e sinais obtidos após aplicação da TWR 

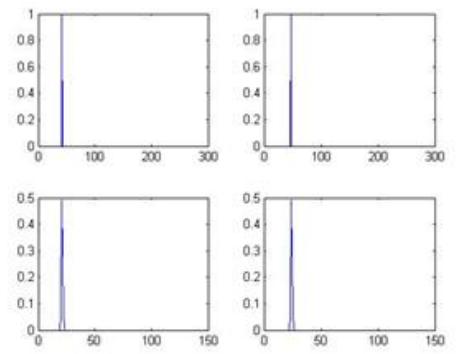

Fig. 5. Sinais Original (Originais), transladado e sinais obtidos após aplicação da TWC

Uma abordagem para implementar a TWC é a chamada TWC de árvore dupla (TWC-AD) que emprega duas Transformadas Wavelets Discretas Reais (TWDR). A primeira determina a parte real e, a segunda, a parte imaginária da TWC-AD, ou seja, $\psi(t)=\psi_{h}(t)+j \psi_{g}(t), j^{2}=-1$.

A TWC-AD-2D é caracterizada por seis Wavelets:

$$
\begin{aligned}
\psi^{1}(x, y) & =\phi(x) \psi(y) \\
\psi^{2}(x, y) & =\psi(x) \psi(y) \\
\psi^{3}(x, y) & =\psi(x) \phi(y) \\
\psi^{4}(x, y) & =\psi(x) \bar{\phi}(y) \\
\psi^{5}(x, y) & =\bar{\psi}(x) \phi(y) \\
\psi^{6}(x, y) & =\phi(x) \bar{\psi}(y)
\end{aligned}
$$

onde $\psi(x)=\psi_{h}(x)+j \psi_{g}(x)$ e $\phi(x)=\phi_{h}(x)+$ $j \phi_{g}(x)$ são funções Wavelet e escala em uma dimensão.

Cada nível da Transformada está associada a doze subimagens, seis imagens que correspondem à parte real e seis à parte imaginária da Transformada, cujos coeficientes são correspondentes aos ângulos $-75,-45,-15,75,45,15$ conforme pode ser observado na figura 7 .

A TWC também apresenta uma melhor seletividade direcional do que a TWR conforme pode ser notado nas figuras 9 a e 10 .

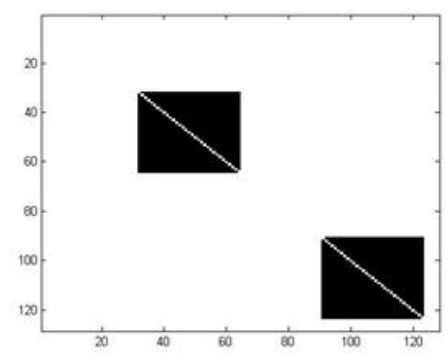

Fig. 6. Imagem Original
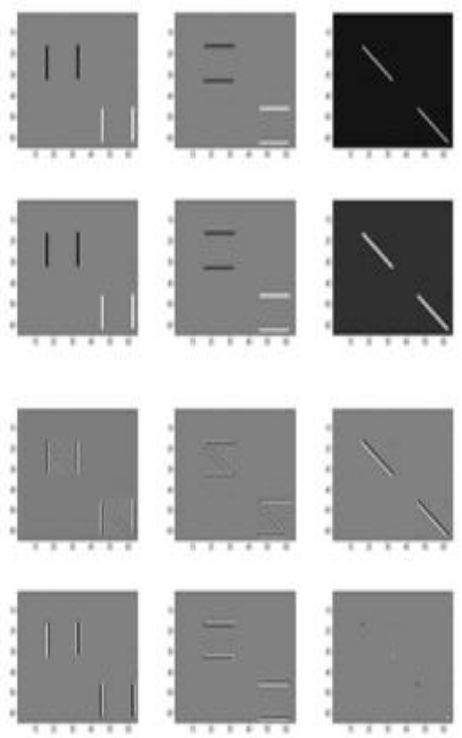

Fig. 7. Subimagens obtidas após aplicação da TWC

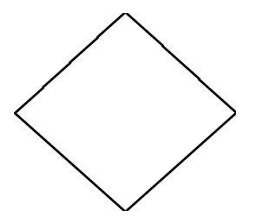

Fig. 8. Imagem Original
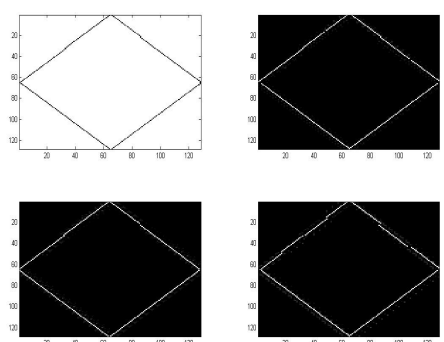

Fig. 9. Subimagens obtidas após aplicação da TWR
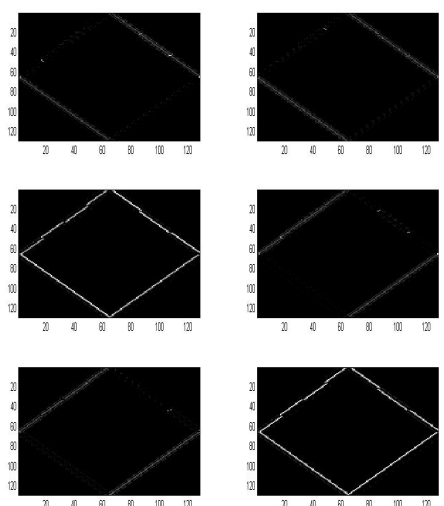

Fig. 10. Subimagens obtidas após aplicação da TWC

A TWC foi utilizada neste trabalho com objetivo de reduzir efeitos indesejáveis causados por 
variação de iluminação, o que poderia comprometer o reconhecimento. Outra característica importante é sua seletividade direcional que permite a detecção de bordas em diferentes resoluções.

\section{Agrupamento Fuzzy}

O agrupamento é uma técnica de classificação não supervisionada de padrões (dados) em grupos (clusters). Intuitivamente, padrões semelhantes devem estar no mesmo grupo e padrões distintos devem estar em grupos diferentes.

O algoritmo c-means (Bezdek, 1981; Bezdek and Dunn, 1975) é utilizado para determinar agrupamentos e seus centros. A relação entre o dado e o agrupamento (centros) é feita através de um grau de pertinência que pode variar em $[0,1]$, em que 0 indica exclusão total desse dado do agrupamento e 1 indica inclusão total do dado no agrupamento. Quanto mais próximo do(s) centro(s) o dado estiver, maior será seu grau de pertinência.

Os dados $X=\left\{x_{k} \mid k=1, \ldots n\right\}$ são classificados minimizando uma função objetivo baseada em uma norma e nos centros dos agrupamentos. A função objetivo é uma soma ponderada dos erros quadráticos entre os grupos que é definida por

$$
J_{m}(X, U, V)=\sum_{k=1}^{n} \sum_{i=1}^{c} \mu_{i k}^{m}\left\|x_{k}-v_{i}\right\|_{A}^{2}
$$

sendo que $1 \leq m \leq \infty$, onde

$$
V=\left\{v_{1}, v_{2}, \ldots, v_{c}\right\}, v_{i} \in R^{n}
$$

é o conjunto dos centros dos agrupamentos e $U=$ $\left(\mu_{i k}\right)$ é uma matriz em que cada elemento $\mu_{i k}$ representa o grau de pertinência de $x_{k}$ no agrupamento $i$.

Se $\left\|x_{k}-v_{i}\right\|_{A}^{2}>0 \forall i, k$ então $U, V$ podem minimizar $J_{m}$ apenas quando $m>1 \mathrm{e}$

$$
\begin{gathered}
v_{i}=\frac{\sum_{k=1}^{n}\left(\mu_{i k}\right)^{m} x_{k}}{\sum_{k=1}^{n}\left(\mu_{i k}\right)^{m}}, \quad 1 \leq i \leq c, \\
\mu_{i k}=\frac{1}{\sum_{j=1}^{c}\left(\frac{\left\|x_{k}-v_{i}\right\|_{A}^{2}}{\left\|x_{k}-v_{j}\right\|_{A}^{2}}\right)^{\frac{1}{m-1}}}
\end{gathered}
$$

$1 \leq i \leq c$ e $1 \leq k \leq n$.

O algoritmo c-means foi utilizado para se determinar os centros de cada classe (ou grupo) que, posteriormente, foram usados no cálculo da função de pertinência $\mu_{i k}$.

\subsection{Integral Fuzzy}

Integrais Fuzzy (Michel Grabisch and Sugeno, 2000; Kwak and Pedrycz, 2004) são funcionais não-lineares definidos com relação a uma Medida Fuzzy. Um conjunto de funções:

$$
g: P(Y) \rightarrow[0,1]
$$

$(P(Y)$ denota a família de todos os subconjuntos de $Y$ ) é chamado uma Medida Fuzzy se as seguintes condições forem satisfeitas:

1. $g(\emptyset)=0, g(Y)=1$;

2. $g(A) \leq g(B), A \subset B, \forall A, B \in P(Y)$;

3. $\lim _{i \rightarrow \infty} g\left(A_{i}\right)=g\left(\lim _{i \rightarrow \infty} A_{i}\right)$.

se $\left\{A_{i}\right\}_{i=1}^{\infty}$ é uma sequência de conjuntos mensuráveis, uma Medida $g_{\lambda}$ Fuzzy satisfaz a propriedade adicional:

$$
g(A \cup B)=g(A)+g(B)+\lambda g(A) g(B)
$$

para todo $A, B \subset Y, A \cap B=\emptyset$ e para algum $\lambda>-1$.

O valor de $\lambda$ pode ser determinado a partir da condição $g(\lambda)=1$, resolvendo

$$
g_{\lambda}(Y)=\frac{1}{\lambda}\left(\prod_{i=1}^{n}\left(1+\lambda g^{i}\right)-1\right), \lambda \neq 0
$$

onde $\lambda \in(-1, \infty)$ e $g^{i}$ é o valor da função densidade. $\mathrm{O}$ cálculo da Integral Fuzzy com relação à Medida $g_{\lambda}$-Fuzzy com os valores da função densidade $g^{i}$. Estes valores podem ser interpretados como grau de importância de cada um dos classificadores.

De acordo com (Kwak and Pedrycz, 2004), a Integral Fuzzy da função $h$ calculada sobre $Y$ com relação à Medida Fuzzy $g$ é definida por:

$\int_{Y} h(y) \circ g()=.\sup _{\alpha \in[0,1]}[\min [\alpha, g(\{y \mid h(y) \geq \alpha\})]]$.

Quando os valores de $h($.$) são ordenados em$ uma sequência decrescente, a Integral Fuzzy de Sugeno é calculada como segue abaixo:

$$
\int_{Y} h(y) \circ g(.)=\max _{i=1}^{n}\left[\min \left(h\left(y_{i}\right), g\left(A_{i}\right)\right)\right] .
$$

onde $A_{i}=\left\{y_{1}, \ldots, y_{n}\right\}$ denota um subconjunto de elementos do universo de discurso.

Os valores de $g\left(A_{i}\right)$ assumidos pela Medida Fuzzy sobre o subconjunto de elementos correspondentes é determinado por:

1. $g\left(A_{1}\right)=g\left(\left\{y_{1}\right\}\right)=g^{1}$

2. $g\left(A_{i}\right)=g^{i}+g\left(A_{i-1}\right)+\lambda g^{i} g\left(A_{i-1}\right), \quad 1 \leq i \leq$ $n$.

A equação (10) não verifica a propriedade de extensão da Integral de Lebesgue, isto é, quando a medida é aditiva a expressão acima não retorna à integral no sentido de Lebesgue. 
Para contornar isso, (Toshiaki Murofushi, 1988) propuseram a chamada Integral de Choquet calculada da seguinte maneira:

$$
\int_{Y} h(y) d g(.)=\sum_{i=1}^{n}\left[h\left(y_{i}\right)-h\left(y_{i+1}\right)\right] g\left(A_{i}\right) h\left(y_{n+1}\right) \text {. }
$$

onde $A_{i}=\left\{y_{1}, \ldots, y_{n}\right\}$ denota um subconjunto de elementos do universo de discurso.

No algoritmo proposto, os valores $h\left(y_{i k}\right)$ da classe $k$ no classificador $i$ são calculados da seguinte forma:

$$
h\left(y_{i k}\right)=\sum_{\mu_{i j} \in C_{k}}\left(\mu_{i j}\right) / N_{k} .
$$

Os valores de $g^{i}$ foram obtidos a partir de

$$
g^{i}=\frac{w_{i} p_{i}}{\cdot \sum_{i} p_{i}}
$$

onde $p_{i}$ denota a taxa de reconhecimento de cada classificador para um conjunto de treinamento, os valores $w_{1}, w_{2}, \ldots, w_{3}$ são pesos associados a cada classificador e foram obtidos experimentalmente.

\section{Algoritmo Proposto}

O algoritmo proposto para Reconhecimento de Faces Humanas baseado na Transformada Wavelet Complexa, PCA, Agrupamento Fuzzy e Integral Fuzzy divide-se em dois estágios:

\subsection{Estágio 1: Treinamento}

Primeiro são selecionadas $C$ imagens de cada classe (pessoa) e, se a base de imagens possui $k$ classes, são selecionadas $C k$ imagens. A cada imagem selecionada é aplicada Transformada Wavelet Complexa, obtendo-se doze subimagens $I 1, I 2, \ldots, I 12$. Seja $C I 1$ o conjunto as subimagens $I 1, C I 2$ o conjunto das subimagens $I 2$ e assim por diante. Depois de concatenar cada uma das matrizes associadas às subimagens obtidas na etapa anterior, é aplicada a PCA a cada um dos conjuntos $C I 1, \ldots, C I 12$, obtendo-se os subespaços $S 1, S 2, \ldots, S 12$. Na última etapa, o algoritmo cmeans é aplicado a cada um dos subespaços gerados anteriormente, obtendo-se os centros de cada grupo.

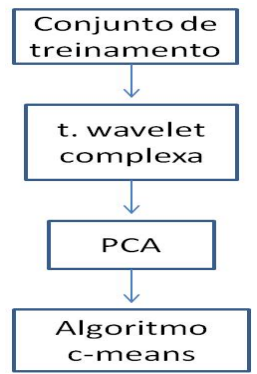

Fig. 11. Estágio 1

\subsection{Estágio 2: Teste}

Após a escolha da imagem que será submetida ao reconhecimento, aplica-se a Transformada Wavelet Complexa, obtendo-se doze subimagens $T 1, T 2, \ldots, T 12$. Cada subimagem é projetada no subespaço correspondente, isto é, $T 1$ é projetada em $S 1, T 2$ em $S 2$ e assim sucessivamente. O algoritmo c-means fornece a função de pertinência associada ao vetor em cada uma das classes e em cada subespaço. A Integral Fuzzy é responsável pela fusão das funções de pertinência.

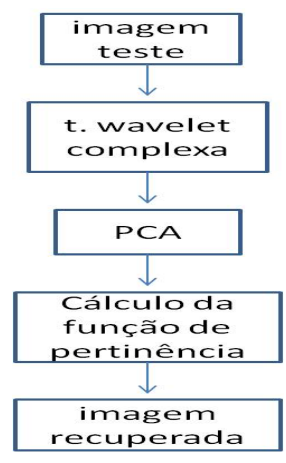

Fig. 12. Estágio 2

\section{Resultados Experimentais}

O algoritmo proposto foi implementado utilizando-se o software Matlab 7.6 (R2008a)@, em um notebook LG, Intel Core i5, com 4G de memória RAM.

Foram realizados testes utilizando-se 165 (BD) imagens da base Yale, divididas em 15 classes, com 11 imagens cada base. Na figura 13, tem-se uma amostra da base BD. As imagens da base foram redimensionadas para a resolução $128 \times 128$.

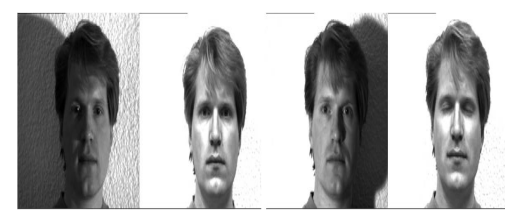

Fig. 13. Base de imagens utilizada

No algoritmo proposto, para cada imagem submetida ao reconhecimento, primeiro é aplicada a TWC, que gera as subimagens que serão projetadas no subespaço PCA; e para cada vetor, no subespaço PCA, é calculado o grau de pertinência em relação aos agrupamentos. Por fim, é realizada a fusão dos classificadores. Haverá sucesso se a imagem submetida ao reconhecimento e a imagem recuperada forem do mesmo indivíduo. Caso contrário, o reconhecimento terá falhado.

Os resultados na tarefa de Reconhecimento de Faces Humanas foram obtidos selecionando alguns parâmetros como a dimensão do subespaço gerado pela PCA (d), a resolução da imagem obtida após aplicação da Transformada Wavelet (r), o número 
de centros de cada agrupamento (c) e o número de imagens do conjunto de treinamento $(\mathrm{C})$.

Na tabela, 1 os parâmetros utilizados foram $d=20, r=32 \times 32, c=2, C=4,5,6$ e, dessa forma foram submetidas ao reconhecimento $7,6,5$ imagens. $\mathrm{O}$ algoritmo proposto é comparado com outras técnicas Transformada Wavelet (achei meio confusa essa parte, maninho...) (TW) + PCA, +ICA e +LDA, em todos os casos a distância utilizada foi a euclidiana. Já na tabela 2 , temos uma comparação do algoritmo proposto com algoritmos utilizando cada uma das doze subimagens obtidas após a aplicação da TWC $d=20$, $r=16 \times 16, c=2$ e $C=3,4,5,6$.

Tabela. 1.Taxa de Reconhecimento

\begin{tabular}{|c||c|c|c|}
\hline \multicolumn{1}{|c||}{ algoritmo } & \multicolumn{3}{c|}{ Conjunto de Treinamento } \\
\hline & 3 & 4 & 5 \\
\hline \hline TW + PCA & $45 \%$ & $63,8 \%$ & $58,8 \%$ \\
\hline TW + ICA & $60,8 \%$ & $67,6 \%$ & $73,3 \%$ \\
\hline TW + LDA & $61,2 \%$ & $77,5 \%$ & $84,8 \%$ \\
\hline proposto & $76,6 \%$ & $86,6 \%$ & $91,1 \%$ \\
\hline
\end{tabular}

Tabela. 2. Taxa de Reconhecimento

\begin{tabular}{|c|c|c|c|c|}
\hline subimagem & \multicolumn{4}{|c|}{ Conjunto de Treinamento } \\
\hline utilizada & 3 & 4 & 5 & 6 \\
\hline 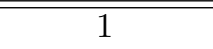 & $70 \%$ & $78 \%$ & $78,8 \%$ & $80 \%$ \\
\hline 2 & $68,3 \%$ & $81,9 \%$ & $80 \%$ & $84 \%$ \\
\hline 3 & $71 \%$ & $76,1 \%$ & $75,5 \%$ & $76 \%$ \\
\hline 4 & $60 \%$ & $65,7 \%$ & $72,2 \%$ & $72 \%$ \\
\hline 5 & $73,3 \%$ & $81,9 \%$ & $85,5 \%$ & $85,3 \%$ \\
\hline 6 & $55,8 \%$ & $63,8 \%$ & $74,4 \%$ & $72 \%$ \\
\hline 7 & $75 \%$ & $71,4 \%$ & $68,8 \%$ & $73,3 \%$ \\
\hline 8 & $71,6 \%$ & $73,3 \%$ & $88,8 \%$ & $82,6 \%$ \\
\hline 9 & $65 \%$ & $72,3 \%$ & $74,4 \%$ & $80 \%$ \\
\hline 10 & $63,3 \%$ & $68,5 \%$ & $74,4 \%$ & $77,3 \%$ \\
\hline 11 & $72,5 \%$ & $81,9 \%$ & $85,5 \%$ & $82,6 \%$ \\
\hline 12 & $66,6 \%$ & $63,8 \%$ & $75,5 \%$ & $72 \%$ \\
\hline máximo & $75 \%$ & $81,9 \%$ & $88,8 \%$ & $85,3 \%$ \\
\hline alg. proposto & $78,3 \%$ & $86 \%$ & $91,1 \%$ & $86,6 \%$ \\
\hline
\end{tabular}

\section{Conclusão}

O reconhecimento de faces humanas é dividido em três partes: aquisição dos dados, extração de características e tomada de decisão. Para extração de características, o algoritmo proposto utiliza a decomposicão da imagem através da Transformada Wavelet Complexa e a PCA. Já na tomada de decisão utiliza a função de pertinência obtida a partir do algoritmo c-means Fuzzy e a Integral Fuzzy para fusão dos classificadores. Concluímos que, independente da subimagem utilizada no reconhecimento de faces humanas, todos os testes realizados demonstram que o algoritmo proposto é sempre superior aos classificadores que utilizam as subimagens individualmente. Ele também se mostrou superior às técnicas $\mathrm{TW}+\mathrm{PCA}$,
TW+ICA e TW+LDA, conforme pode ser observado na tabela 1 .

\section{Referências}

Bezdek, J. C. (1981). Pattern Recognition with Fuzzy Objective Function Algorithms, Plenum Press. DOI: 10.1007/978-1-4757-0450-1

Bezdek, J. C. and Dunn, J. C. (1975). Optimal Fuzzy Partitions: A heuristic for estimating the parameters in a mixture of normal distributions, IEEE Transactions on Computers. DOI: 10.1109/T-C.1975.224317

Bruce A. Draper, Kyungim Baek, M. S. and Bevedge, J. R. (2003). Recognition faces with $P C A$ and $I C A$, Computer Vision and Image Understanding.

Goswami, J. C. and Chan, A. K. (2011). Fundamentals of Wavelets: Theory, Algorithms and Applications, Wiley. DOI: 10.1002/9780470926994

Ivan W. Selesnick, R. G. B. and Kingsbury, N. G. (2005). The Dual-Tree Complex Wavelet Transform, IEEE Signal Processing Magazine.

Kwak, K.-C. and Pedrycz, W. (2004). Face Recognition Using Fuzzy Integral and Wavelet Decomoposition Method, IEEE Tansaction on Systems.

Kyung-A Kim, S. Y. O. and Choi, H.-C. (2004). Facial Feature Extraction Using PCA and Wavelet Multi-Resolution Image, Proceedings of the 6th IEEE International Conference on Automatic Face and Gesture Recognition.

iu, C.-C. and Dai, D.-Q. (2009). Face Recognition Using Dual-Tree Complex Wavelet Features, IEEE Transactions on Image Processing.

Martinez, A. M. and Kak, A. C. (2001). PCA versus $L D A$, IEEE Transactions on Pattern Analysis and Machine Intelligence.

Michel Grabisch, T. M. and Sugeno, M. (2000). Fuzzy Measures and Integrals: Theory and Applications, Phy-Verlag. DOI: 10.1109/34.908974

P.C.Yuen, D. and G.C.Feng (1998). Wavelet based PCA for Human Face Recognition, Image Analysis and Interpretation,IEEE Southwest Symposium.

Toshiaki Murofushi, M. S. (1988). Measures and the Choquet Integral as an integral with respect to a Fuzzy Measure, Fuzzy Sets Syst.

Water, J. S. (1999). A Primer on Wavelets and Their Scientific Applications, Chapman e Hall/CRC. 